\title{
Composição química e produtividade dos principais componentes do óleo essencial de Baccharis dracunculifolia DC. em função da adubação orgânica
}

\author{
SANTOS, R.F. ${ }^{1 *}$; ISOBE, M.T.C. ${ }^{1}$; LALLA, J.G. ${ }^{1}$; HABER,L.L. ${ }^{2}$; MARQUES, M.O.M. ${ }^{2}$; MING, L.C. ${ }^{1}$ \\ ${ }^{1}$ UNESP/FCA - Campus Botucatu, Departamento de Produção Vegetal, Setor Horticultua. Rua José Barbosa de \\ Barros, 1780, CEP: 18610-307, Botucatu-Brasil '2Instituto Agronômico de Campinas. Avenida Barão de Itapura, \\ 1481, Caixa Postal 28, CEP: 13012-970, Campinas-Brasil *rosafs1208@yahoo.com.br
}

\begin{abstract}
RESUMO: Objetivou-se avaliar a composição química e produtividade dos principais componentes do óleo essencial de Baccharis dracunculifolia DC. em função de doses de composto orgânico $\left(0,10,20,30,40\right.$ e $\left.50 \mathrm{t} \mathrm{ha}^{-1}\right)$. Foi realizada uma colheita, aos 150 dias após o transplante das mudas. O óleo essencial, da massa seca útil da parte aérea, foi extraído por hidrodestilação e analisado em cromatógrafo a gás acoplado a espectrômetro de massas (Shimadzu, QP-5000). A identificação dos constituintes químicos foi realizada através da análise comparativa dos espectros de massas das substâncias com o banco de dados do sistema CG-EM (Nist 62.lib), literatura e índice de retenção. Os resultados foram submetidos à análise de variância pelo teste $F$, às médias obtidas foram submetidas à análise de regressão e o teste Tukey para o efeito das doses de composto orgânico. Os três componentes sesquiterpênicos, E-nerolidol, espatulenol e óxido de cariofileno, perfazem $58,44 \%$ da média relativa da composição química do óleo essencial de $B$. dracunculifolia, composto pela presença de 28 substâncias. Na produtividade dos componentes $\gamma$-muroleno, valenceno, $\delta$-cadineno e $E$-nerolidol as dosagens estudadas influenciaram as plantas, que na dosagem $30 \mathrm{t} \mathrm{ha}^{-1}$ obtiveram os melhores resultados. Se o objetivo no cultivo de $B$. dracunculifolia for o componente espatulenol as dosagens 30 e $40 \mathrm{t} \mathrm{ha}^{-1}$ obtiveram os melhores resultados. Para a produtividade do componente óxido de cariofileno as dosagens estudadas influenciaram as plantas, que na dosagem $40 \mathrm{t} \mathrm{ha}^{-1}$ obtiveram os melhores resultados.
\end{abstract}

Palavras-chave: Alecrim-do-campo, composto orgânico, plantas medicinais, composição química

ABSTRACT: Chemical composition and productivity of essential oil of Baccharis dracunculifolia DC. as affected by organic compound. The objective was to evaluate the chemical composition and productivity of the main components of the essential oil of Baccharis dracunculifolia DC. due to organic compound $\left(0,10,20,30,40\right.$ and $\left.50 \mathrm{t} \mathrm{ha}^{-1}\right)$. We performed a harvest, 150 days after transplanting. The essential oil of the dry mass useful in the aerial part was extracted by hydrodistillation and analyzed in gas chromatograph coupled to a mass spectrometer (Shimadzu, QP-5000). The identification of chemical constituents was performed by comparative analysis of mass spectra of substances with the database of the GC-MS (Nist 62.lib), literature and retention rates. The results were subjected to analysis of variance by $F$ test, the averages obtained were subjected to regression analysis and Tukey test for the effect of doses of organic compound. The three components sesquiterpene, E-nerolidol, espatulenol and caryophyllene oxide, account for $58.44 \%$ of the average for the chemical composition of essential oil of $B$. dracunculifolia, composed by the presence of 28 substances. Productivity of components $\gamma$-murolene, valencene, $\delta$-cadinene and $E$-nerolidol was influenced by doses, which in dose $30 \mathrm{t}$ ha $^{-1}$ obtained the best results. If the goal in the cultivation of $B$. dracunculifolia is the component espatulenol dosages 30 and $40 \mathrm{tha}^{-1}$ obtained the best results. To the productivity of caryophyllene oxide doses studied influenced the plants, which in dose $40 \mathrm{t} \mathrm{ha}^{-1}$ obtained the best results.

Key words: Alecrim-do-campo, organic compound, medicinal plants, chemical composition

Recebido para publicação: setembro de 2011

Aceito para publicação: março de 2012

Rev. Bras. PI. Med., Botucatu, v.14, n.esp., p.224-234, 2012. 


\section{INTRODUÇÃO}

A produção de biomassa e princípios ativos nas plantas medicinais, aromáticas e condimentares depende de vários fatores dentre eles o manejo fitotécnico. Dentre os insumos que maximizam a produção das plantas, o adubo é um dos responsáveis pela elevação da produtividade e qualidade dos produtos obtidos. As plantas medicinais e aromáticas também dependem de suprimento adequado de nutrientes para boas produtividades agrícolas. Mesmo com todos os esforços para eficiente nutrição, que começa com a análise química do solo e correção da acidez, quando necessário e fornecimento adequado de nutrientes, as plantas podem ficar sujeitas a condições de disponibilidade sub ou supraótima de nutrientes. Os limites da faixa de concentração considerada adequada variam amplamente com vários fatores como elemento mineral, genótipo, órgão, etc., podendo ser mais estreita ou mais larga. O suprimento inadequado de um nutriente essencial, por deficiência ou excesso, além de modificações no metabolismo celular, crescimento, desenvolvimento e produtividade, pode se manifestar por meio de sintomas visuais, sendo muitas vezes assim identificado. Aadubação orgânica é fonte de nutrientes para as plantas além de fornecer suprimento adequado, contribui para a melhoria da estrutura física do solo o aumento de retenção de água, diminuindo perdas por erosão, favorecendo o controle biológico devido à maior população microbiana e melhorando a capacidade tampão do solo.

A espécie Baccharis dracunculifolia DC. é popularmente conhecida como vassoura, vossourinha ou alecrim-do-campo, e amplamente utilizada na medicina tradicional. É planta arbustiva, lenhosa, perene que ocorre no Brasil, de Minas Gerais ao Rio Grande do Sul, nos países do Mercosul e nos vales elevados da Bolívia e é comum em campos abertos, pastagens abandonadas e áreas de sucessão. $O$ arbusto cresce em média 2 a 3 metros. É espécie dióica, que se reproduz por sementes (aquênios), com caules bastante ramificados, sendo estes ramificados e densamente recobertos por tricomas.

$B$. dracunculifolia destaca-se por se associar a grande número de insetos herbívoros, tendo sido registradas 90 morfo-espécies presentes em 29 plantas femininas e 43 masculinas, incluindo o indutor de galha Neopelma baccharidis (Araújo et al., 1995; Colevatti \& Sperber, 1997; Espírito-Santo et al., 1999). Devido à intensidade de frequência de herbivoria, que ocorre em indivíduos de $B$. dracunculifolia, os mecanismos de defesa química dessa planta têm sido alvos de diversos estudos.

Em estudo químico de $B$. dracunculifolia (Costa, 2009) foram identificados monoterpenos, $\beta$-pineno, limoneno e terpinen-4-ol, os principais sesquiterpenos hidrocarbonetos, $\beta$-cariofileno, $\alpha$-humuleno, germacreno $D$, trans- $\beta$-guaieno e $\delta$ cadineno. Os sesquiterpenos oxigenados $E$-nerolidol e espatulenol se encontraram como constituintes majoritários (Queiroga, 1989). A mesma autora relata que no estudo fitoquímico do óleo essencial de $B$. dracunculifolia identificou 55 compostos, e mais dois compostos inéditos na literatura: $1 \mathrm{H}$-cicloprop[e] azuleno, 1a,2,3,4, 4a,5 7 a, 7b-octa-hidro-1,1,4, 7tetrametil-(1aa, 4b, 4aa, 7 ab, 7 ba) 56 e $1 \mathrm{H}$ cicloprop[e] azuleno, 1a,2,3,4, 4a, 5,7a,7b-octa-hidro1,1,4 7-tetrametil- (1aa,4a,4aa,7ab,7ba) 57. Em estudos sobre os constituintes das folhas de $B$. dracunculifolia e sua atividade citotóxica, (Fukuda et al., 2006) descobriram que os extratos alcoólicos destas folhas apresentam atividade citotóxica potente contra células de leucemia e também descreveram o isolamento e elucidação estrutural de dois novos compostos, baccharisketone e p-methoxythymol acetato.

O óleo essencial da B. dracunculifolia DC. é considerado essência exótica sendo exportada como matéria-prima para confecção de perfumes e tem como componentes majoritários os sesquiterpenos E-nerolidol e espatulenol (Queiroga, 1989).

O E-nerolidol é um sesquiterpeno presente nos óleos essenciais de diversas plantas e foi aprovado nos Estados Unidos da América pela agência reguladora Food and Drug Administration (FDA) como um agente flavorizante utilizado em alimentos (Arruda et al., 2005). Segundo (Wattenberg, 1991) apud (Arruda et al., 2005), esse composto também apresenta propriedade antineoplásica. Estudos sobre esse composto indicam o efeito inibidor do crescimento do Plasmodium falciparum, o agente causador da malária (Macedo et al., 2002) e de Leishmania amazonensis, causador da leishmaniose tegumentar americana (Arruda et al., 2005).

O componente espatulenol apresenta atividade biológica importante com propriedades antibacterianas e moderada atividade citotóxica (Limberger et al., 2004).

Outro componente, porém não majoritário, do óleo essencial de $B$. dracunculifolia é o sesquiterpeno óxido de cariofileno, que a literatura relata possuir atividade anticarcinogênica (Zheng et al., 1992).

Dentre os fatores que podem interferir nos princípios ativos de plantas, a nutrição é um dos que requerem maior atenção, pois o excesso ou a deficiência de nutrientes podem estar diretamente correlacionados à variação na produção de substâncias ativas. De acordo com a quantidade exigida pelas plantas, os nutrientes são conhecidos como macro (N, P, K Ca e Mg) e micronutrientes (B, $\mathrm{Cl}, \mathrm{Cu}, \mathrm{Fe}, \mathrm{Mn}, \mathrm{Mo} \mathrm{e} \mathrm{Ni)}$; sendo os macronutrientes

Rev. Bras. PI. Med., Botucatu, v.14, n.esp., p.224-234, 2012. 
$\mathrm{N}$, P e K, de modo geral, utilizados em maior proporção na adubação. Esta estreita relação soloplanta sem dúvida interfere nos passos metabólicos que originam substâncias bioativas. O cultivo quando mal conduzido pode gerar plantas medicinais com produtos ativos em quantidades pequenas, tornando a comercialização inviável ou de maneira oposta, incrementar a quantidade de substâncias consideradas tóxicas, tornando o produto nocivo e, portanto, de utilização terapêutica inviável.

Considerando estes fatores o objetivo deste trabalho foi avaliar a composição química e a produtividade dos principais componentes do óleo essencial de Baccharis dracunculifolia DC., em função da adubação orgânica.

\section{MATERIAL E MÉTODO}

O experimento foi conduzido no pomar experimental do Departamento de Produção Vegetal Horticultura da Faculdade de Ciências AgronômicasUNESP, Campus de Botucatu/SP, que apresenta as coordenadas geográficas: $22^{\circ} 55^{\prime} 5^{\prime \prime} \mathrm{S}, 48^{\circ} 26^{\prime} 22^{\prime \prime O}$ e altitude $810 \mathrm{~m}$. O tipo climático predominante no local é o temperado quente (mesotérmico) com chuvas no verão e seca no inverno (Cwa - Koppen), tendo temperatura média anual de $20,5^{\circ} \mathrm{C}$ e precipitação pluviométrica média anual de $1533 \mathrm{~mm}$ (Cunha et al., 1999). O solo da área foi classificado como Nitossolo Vermelho, segundo os critérios da Embrapa (2006). Conforme análise de solo realizada previamente, foi corrigida $\mathrm{V} \%$ para $70 \%$ por meio da aplicação de calcário Fuller PRNT 100\%, sendo utilizado $2 \mathrm{t} \mathrm{ha}^{-1}$ deste calcário. O solo foi arado e gradeado para o plantio. A análise química do solo foi realizada de acordo com a instrução normativa DAS 28 de 27/07//2007, Mapa (2007).

Utilizou-se mudas de alecrim do campo produzidas por sementes, de população espontânea de Botucatu (São Paulo) (S $22^{\circ} 50^{\prime} 54,0^{\prime \prime}$; WO $48^{\circ}$ 26 ' $04,2 ")$, coletadas em maio de 2009. As plantas foram identificadas e as exsicatas foram depositadas no Herbário do Instituto de Biociências da UNESP de Botucatu, sob o número BOTU 027340 para as plantas femininas e BOTU 027341 para as plantas masculinas. Após a coleta as inflorescências foram colocadas em bandejas plásticas e secas à sombra, em temperatura ambiente (média de $22^{\circ} \mathrm{C}$ ), e submetidas à debulha manual para a separação das sementes. As sementes foram colocadas para germinar em tubetes cilindro-cônicos de polietileno com volume de $50 \mathrm{~cm}^{3}$, preenchidos com substrato comercial Carolina Soil ${ }^{\circledR}$, mantidas sob casa-devegetação com luminosidade de $50 \%$, sendo irrigadas diariamente por nebulização intermitente a cada quinze minutos até o transplantio das mudas para o campo. O plantio definitivo foi realizado em 5 de fevereiro de 2010, em covas, no espaçamento de 1,0 x $1,0 \mathrm{~m}$, entre plantas e linhas, com irrigação por gotejamento, aos 84 dias após a semeadura (DAS), em decorrência de condições climáticas desfavoráveis, para a implantação com 60 dias após a semeadura (DAS), conforme planejamento inicial. No plantio o composto orgânico foi misturado com a terra retirada para a abertura da cova e esta mistura foi colocada na cova de plantio. A área de cultivo foi conduzida dentro dos critérios de boas práticas agrícolas sendo mantida limpa de invasoras por capina manual e com roçadeiras.

O delineamento experimental utilizado foi em blocos ao acaso, com seis tratamentos (doses de composto orgânico foram de $0,10,20,30,40$ e 50 tha $^{1}$ ), com quatro repetições.

Cada parcela foi constituída por 12 plantas úteis, totalizando 288 plantas úteis. Foram plantadas mudas de alecrim do campo, também em volta de cada parcela, ou seja, plantou-se 332 mudas de alecrim do campo ao redor o experimento como bordadura, totalizando 620 plantas, numa área de $620 \mathrm{~m}^{2}$.

A análise química do composto foi realizada de acordo com metodologia descrita por Raij (2001). O composto orgânico apresentou as características químicas, descritas na Tabela1.

A matéria prima, resíduos de origem vegetal e animal para o experimento de compostagem foi obtida junto à Centroflora Anidro do Brasil e ao

TABELA 1. Resultado da análise química do composto orgânico utilizado na adubação do alecrim-do-campo, 2010.

\begin{tabular}{|c|c|c|c|c|c|c|c|c|c|c|c|c|c|}
\hline \multirow{3}{*}{$\begin{array}{l}\text { M.O. } \\
(\%)\end{array}$} & \multirow{3}{*}{$\mathrm{C} / \mathrm{N}$} & \multirow{3}{*}{$\begin{array}{l}\text { pH } \\
\mathrm{H}_{2} \mathrm{O}\end{array}$} & \multicolumn{11}{|c|}{ Nutrientes } \\
\hline & & & $\mathbf{N}$ & $\mathrm{P}_{2} \mathrm{O}_{5}$ & $\mathrm{~K}_{2} \mathrm{O}$ & $\mathrm{Ca}$ & Mg & $s$ & $\mathrm{Cu}$ & $\mathrm{Zn}$ & $\mathrm{Fe}$ & Mn & UM \\
\hline & & & \multicolumn{5}{|c|}{$\%$ na matéria seca } & & & \multicolumn{4}{|c|}{$\mathrm{mg} \mathrm{kg}^{-1}$ na matéria seca } \\
\hline 37 & $10 / 1$ & 7,3 & 2,3 & 0,43 & 0,19 & 1,8 & 0,2 & 0,2 & 23 & 107 & 7767 & 268 & 4,17 \\
\hline
\end{tabular}

Fonte: Departamento de Recursos Naturais/Ciência do solo/Laboratório de Fertilidade e Corretivos/UNESP-FCA Campus de Botucatu-SP. ${ }^{1}$ Composto orgânico oriundos de mistura das seguintes espécies 1 - Pilocarpus microphyllus Stapf ex Wardleworth, 2 - Aesculus hippocastanum L., 3 - Atropa belladona L., 4 - Trichilia emarginata (Turcz.) C. DC., 5 - Passiflora alata Curtis, 6 - Paullinia cupana Kunth.

Rev. Bras. PI. Med., Botucatu, v.14, n.esp., p.224-234, 2012. 
confinamento dos novilhos precoces da UNESP de Botucatu, respectivamente. Os resíduos vegetais da produção de extratos botânicos disponíveis que foram fornecidos pela Centroflora foram oriundos da mistura das seguintes espécies: 1 - Pilocarpus microphyllus Stapf ex Wardleworth, 2 - Aesculus hippocastanum L., 3 - Atropa belladona L., 4 - Trichilia emarginata (Turcz.) C. DC., 5 - Passiflora alata Curtis e 6 Paullinia cupana Kunth. Os resíduos foram secos até eliminação do solvente e montados em pilhas com aproximadamente $5 \mathrm{~m}^{3}$. Esterco de curral foi adicionado a fim de balancear a relação $\mathrm{C} / \mathrm{N}$ para $35 / 1$, em função das análises químicas, objetivando-se finalizar o processo de compostagem com o máximo de $\mathrm{C} / \mathrm{N}$ de 18/1 (Kiehl, 2002). O manejo das pilhas foi conduzido conforme (Kiehl, 1985). Durante o processo de compostagem, avaliou-se três amostras por pilha ao final de 90 dias, quando os compostos já estavam prontos para determinação dos minerais, nitrogênio total, carbono orgânico total e pH, de acordo com Laboratório Nacional de Referência Vegetal (1988). Com base nestes resultados os fertilizantes obtidos foram caracterizados como fertilizante orgânico misto, de acordo com o Art. $2^{\circ}$ classe " $A$ " e enquadrado nos demais itens seguindo a Instrução normativa № 23 , de 31 de agosto de 2005 da Secretaria de defesa agropecuária do Ministério da Agricultura e Abastecimento em acordo com o Decreto n' 5.351, de 21 de janeiro de 2005. A análise química do composto foi realizada de acordo com metodologia descrita por Raij (2001). O composto orgânico apresentou as seguintes características químicas: matéria orgânica $(\%)=37$; relação carbono nitrogênio $\mathrm{C} / \mathrm{N} \mathrm{10/1;} \mathrm{pH}$ em água $=7,3 ; \mathrm{N}, \mathrm{P}_{2} \mathrm{O}_{5}, \mathrm{~K}_{2} \mathrm{O}, \mathrm{Ca}, \mathrm{Mg}$, $\mathrm{S}(\%$ na matéria seca) $=2,3 ; 0,43 ; 0,19 ; 1,8 ; 0,2 ; 0,2$ Cobre, Zinco, Ferro e Manganês, ( $\mathrm{mg} \mathrm{kg}^{-1}$ na matéria seca $)=23 ; 107 ; 7767 ; 268$.

A área de cultivo foi conduzida dentro dos critérios de boas práticas agrícolas sendo mantida limpa de invasoras por capina manual e com roçadeiras. Realizou-se a colheita, das sete às nove horas da manhã, no dia cinco de julho de 2010, 150 dias após o transplante das mudas (DAT). O corte foi feito com tesoura de poda e podão, à $30 \mathrm{~cm}$ do solo. Logo após a colheita, as plantas foram acondicionadas em sacos de papel, devidamente identificadas e colocadas para secagem em estufa de circulação forçada de ar, em temperatura controlada a $40^{\circ} \mathrm{C}$, até a obtenção de massa seca constante.

Determinandou-se a massa seca da parte aérea útil, a qual foi definida como caule de até 2 milímetros de diâmetro, verde e com folhas, por ser esta a parte da planta produtora de óleo essencial.

A extração do óleo essencial foi realizada no Laboratório de Produtos Naturais, do Centro de Pesquisa e Desenvolvimento de Recursos Genéticos Vegetais do Instituto Agronômico de Campinas,
Campinas - SP, de setembro de 2010 a janeiro de 2011 O óleo essencial foi extraído por hidrodestilação, em aparelho tipo Clevenger. Craveiro et al. (1981), utilizando-se $50 \mathrm{~g}$ de massa seca útil da parte aérea em $2 L$ de água destilada durante duas horas.

A identificação dos constituintes químicos foi realizada através da análise comparativa dos espectros de massas das substâncias com o banco de dados do sistema CG-EM (Nist 62.lib), literatura (Mclafferty \& Stauffer, 1989) e índice de retenção (Adams, 2001). Os índices de retenção de Kovat's (IR) das substâncias foram obtidos através da coinjeção do óleo essencial com uma mistura padrão de n-alcanos (C9H20 - C25H52 Sigma Aldrich, 99\%), aplicando-se a equação de Van den Dool e Kratz (Van Den Dool \& Kratz, 1963). A composição química do óleo essencial foi apresentada em forma de tabela, com a porcentagem relativa das substâncias obtida por normalização de área.

As variáveis analisadas foram composição química do óleo essencial em percentagem relativa e a produtividade dos principais componentes em quilos por hectare. Os resultados foram submetidos à análise de variância pelo teste $F$, às médias obtidas foram submetidas à análise de regressão e o teste Tukey (Banzatto \& Kronka, 1995) para o efeito das doses de composto orgânico.

\section{RESULTADO E DISCUSSÃO}

$\mathrm{Na}$ análise cromatográfica do óleo essencial de $B$. dracunculifolia, detectou a presença de 28 substâncias, (Tabela 2), sendo estas as identificadas: $\alpha$-pineno, $\beta$-pineno, mirceno, limoneno, E-pinocarveol, mirtenol, $E$-cariofileno, $\beta$-santaleno, oxido de cabreuva $B, \gamma$-muuroleno, valenceno, $\delta$-cadineno, isômero de nerolidol, $E$-nerolidol, espatulenol, óxido de cariofileno, globulol, khusimona, $\beta$-oplopenona, epi- $\alpha$-cadinol, $\beta$ murolol, cubenol, 14-hidroxi-(z)-cariofileno e eudesma4(15),7-dien-1 $\beta$-ol, com predominância dos sequisterpenos $E$-nerolidol e espatulenol, (Figura 1) com porcentagem relativa média de $33,81 \%$ e $18,96 \%$, respectivamente (Tabela 2). Os vários compostos presentes no óleo essencial de alecrim-do-campo, (B. dracunculifolia) como o E-nerolidol, espatulenol, $\delta$-cadineno, $\beta$-pineno, globulol entre outros mostram que os compostos identificados neste trabalho estão de acordo com a literatura (Loayza et al., 1995; Ferracini et al., 1995; Queiroga, 1989).

Os constituintes químicos do óleo essencial das folhas (Tabela 2) demonstram que o alecrim-docampo tem no metabolismo secundário, a presença da via biossintética do mevalonato (Simões, 2007), da qual se originaram os terpenóides. Há pelo menos, uma via citosólica ou rota do mevalonato (MVA) e outra plastidial ou rota 5 -fosfato de 1-desoxi-D-xilulose (DXP). Ambas conduzem à produção de isômeros de

Rev. Bras. PI. Med., Botucatu, v.14, n.esp., p.224-234, 2012. 


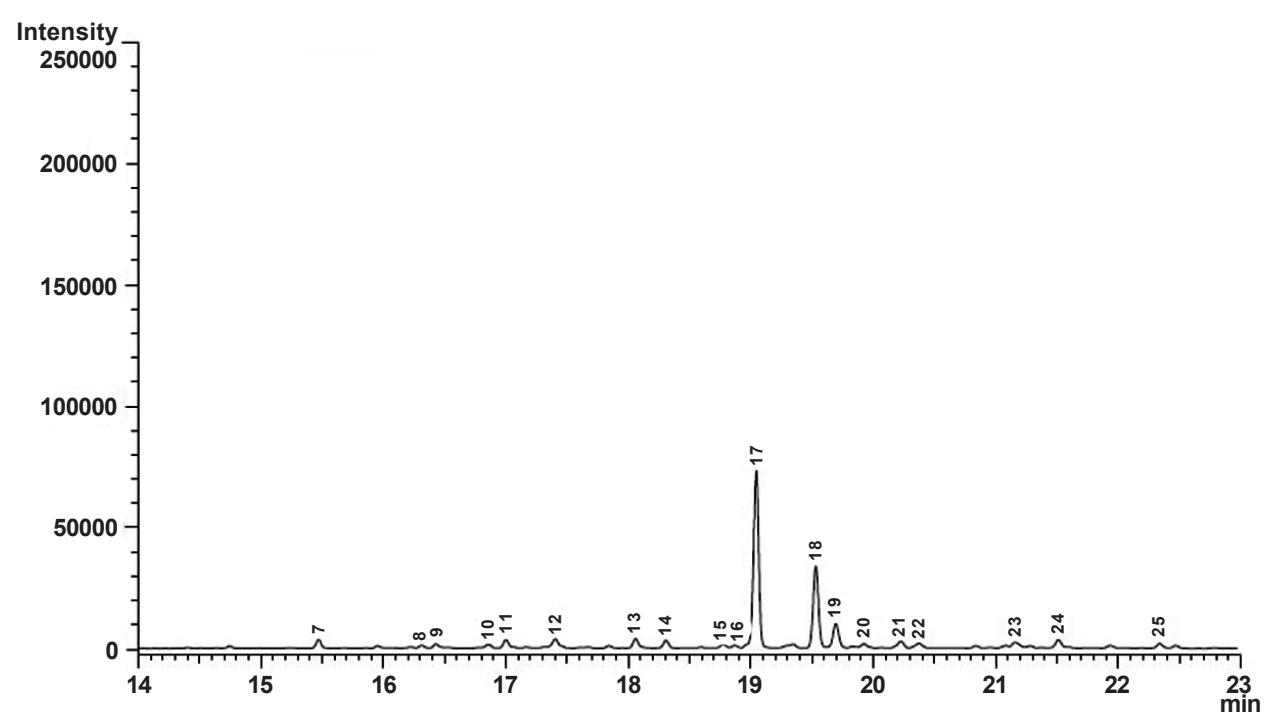

FIGURA 1. Cromatograma de Íons Totais (CG-EM) e composição das substâncias majoritárias, sendo 17: Enerolidol, 18: espatulenol, presentes no óleo essencial de folhas de $B$. dracunculifolia avaliada em doses crescentes de composto orgânico: 0, 10, 20, 30, 40 e 50 t ha-1 $^{-1}$ aos 150 DAT (dias após o transplante), cultivadas em Botucatu, SP, 2010.

isopreno, o disfosfato de isoprentenila (IPP) e/ou o difosfato de gama, gama-dimetilalila (DMAPP), os quais constituem os monômeros a partir dos quais se originaram os diferentes terpenóides. A partir da condensação de uma molécula de IPP com DMAPP forma-se o difosfato de geralina (GPP), precursor dos monoterpenóides. A condensação de uma nova molécula de IPP e do GPP leva ao precursor dos sesquiterpenóides, difosfato de farnesila (FPP) (Bouvier et al., 2005) (Dewick, 2002).

Nota-se que não existe tendência uniforme na variação da percentagem média relativa dos metabólitos especializados, em função da doses crescentes de composto orgânico (Tabela 1), visto que as principais substâncias ( $\gamma$-muuroleno, valenceno, $\delta$-cadineno, $E$-nerolidol, espatulenol e óxido de cariofileno) são sesquiterpênicas originadas pela mesma rota metabólica, não havendo priorização para formação de um ou outro metabólito. Os componentes $\gamma$-muuroleno e valenceno obtiveram o melhor resultado na dose $10 \mathrm{t} \mathrm{ha}^{-1}$; já o $\delta$-cadineno obteve o melhor resultado na dose $20 \mathrm{t} \mathrm{ha}^{-1}$; o $E$ nerolidol obteve o melhor na dose $30 \mathrm{t}^{\text {ha }}{ }^{-1} \mathrm{e}$ responderam positivamente ao estress nutricional as substâncias espatulenol e óxido de cariofileno com a maior média para a dose $0 \mathrm{t} \mathrm{ha}^{-1}$.

Os terpenóides, em especial mono e sesquiterpenóides, apresentam diversas funções nos vegetais. Proteção contra herbívoros, agentes microbianos e atração de polinizadores são as funções mais conhecidas. Outras funções ainda pouco elucidadas para a maioria dos derivados isoprênicos, dizem respeito a efeitos de termo-proteção, proteção a danos oxidativos, foto-respiração à altas temperaturas e baixas concentrações de $\mathrm{O}_{2}$, alelopatia e foto-proteção (Owen, 2005). Plantas medicinais oriundas de cultivo orgânico são mais resistentes a pragas e doenças, reduzindo-se a necessidade de controle fitossanitário (Box, 1973). Portanto, a adubação orgânica favorece maior resistência ao ataque de pragas e doenças e isso pode justificar a resposta quadrática observada na produtividade dos principais componentes do óleo essencial de alecrim-do-campo, pois, o decréscimo na produtividade dos principais componentes do óleo essencial, pode ser atribuído como resposta ao equilíbrio nutricional, proporcionado pelo composto orgânico, e assim a planta não precisa de maior produção de compostos para sua defesa.

Para o componente sesquiterpênico $\gamma$ muuroleno (Figura 2) verificou-se diferença estatística significativa para o teste Tukey a $5 \%$ de probabilidade $(p<0,05)$, entre as doses avaliadas (Tabela 2 ) através da análise de regressão observou-se o aumento gradativo na produtividade deste composto com o acréscimo da dose de composto orgânico, sendo que a dose de $30 \mathrm{t} \mathrm{ha}^{-1}$ com $0,22 \mathrm{~kg} \mathrm{ha}^{-1}$, responsável pelo incremento de $0,13 \mathrm{~kg} \mathrm{ha}^{-1}$ em relação à testemunha. $\mathrm{O}$ aumento da adubação a partir desse ponto causou diminuição neste componente.

Compostos sesquiterpênicos possuem atividades como inibidores de germinação e crescimento radicular, sobre diversas espécies vegetais, o que pode viabilizar o desenvolvimento de produtos para o controle de plantas invasoras, e consequentemente tornar o resultado deste trabalho importante.

O sesquiterpeno valenceno apresentou 
TABELA 2. Composição química (\% média relativa), do óleo essencial de $B$. dracunculifolia avaliada em doses crescentes de composto orgânico: 0, 10, 20, 30, 40 e 50 t ha-1 $^{-1}$, aos 150 DAT (dias após o transplante), cultivadas em Botucatu, SP.

\begin{tabular}{|c|c|c|c|c|c|c|c|c|}
\hline \multirow{2}{*}{ Substâncias (\%) } & \multicolumn{6}{|c|}{ Tratamentos (t ha- 1 ) } & \multirow{2}{*}{ Ikcal } & \multirow{2}{*}{ Iklit } \\
\hline & 0 & 10 & 20 & 30 & 40 & 50 & & \\
\hline$\alpha$-pineno & 1,01 & 1,68 & 1,50 & 2,66 & 1,90 & 1,89 & 932 & 939 \\
\hline$\beta$-pineno & 1,93 & 2,00 & 2,70 & 2,50 & 2,57 & 2,80 & 975 & 979 \\
\hline Mirceno & $\operatorname{tr}$ & 0,44 & $\operatorname{tr}$ & 0,43 & 0,32 & 0,34 & 988 & 990 \\
\hline Limoneno & 1,15 & 1,41 & 1,70 & 1,89 & 1,72 & 2,11 & 1026 & 1029 \\
\hline E-pinocarveol & $\operatorname{tr}$ & $\operatorname{tr}$ & $\operatorname{tr}$ & $\operatorname{tr}$ & $\operatorname{tr}$ & $\operatorname{tr}$ & 1135 & 1139 \\
\hline Mirtenol & $\operatorname{tr}$ & $\operatorname{tr}$ & $\operatorname{tr}$ & $\operatorname{tr}$ & $\operatorname{tr}$ & $\operatorname{tr}$ & 1193 & 1195 \\
\hline E-cariofileno & 3,31 & 3,70 & 3,52 & 3,13 & 3,54 & 2,86 & 1417 & 1419 \\
\hline$\beta$-santaleno & $\operatorname{tr}$ & $\operatorname{tr}$ & 0,61 & 0,53 & 0,50 & 0,64 & 1458 & 1459 \\
\hline óxido de cabreuva B & 0,80 & 0,93 & 1,05 & 0,98 & 0,95 & 1,08 & 1474 & 1464 \\
\hline$\gamma$-muuroleno & 5,51 & 6,04 & 5,53 & 5,26 & 4,99 & 4,15 & 1478 & 1477 \\
\hline Valenceno & 5,51 & 5,81 & 5,06 & 4,89 & 4,99 & 4,62 & 1493 & 1496 \\
\hline$\delta$-cadineno & 3,64 & 3,81 & 4,21 & 3,67 & 3,50 & 3,53 & 1520 & 1523 \\
\hline N.I. * & 0,92 & 1,00 & 1,21 & 1,11 & 1,25 & 1,74 & 1531 & 1529 \\
\hline N.I.* & 0,48 & $\operatorname{tr}$ & $\operatorname{tr}$ & $\operatorname{tr}$ & $\operatorname{tr}$ & $\operatorname{tr}$ & 1551 & 1561 \\
\hline isômero de nerolidol & $\operatorname{tr}$ & $\operatorname{tr}$ & $\operatorname{tr}$ & $\operatorname{tr}$ & $\operatorname{tr}$ & $\operatorname{tr}$ & 1558 & 1563 \\
\hline E-nerolidol & 32,06 & 32,40 & 33,09 & 37,57 & 34,19 & 33,52 & 1563 & 1563 \\
\hline Espatulenol & 21,65 & 19,20 & 17,99 & 17,17 & 18,40 & 19,34 & 1576 & 1567 \\
\hline óxido de cariofileno & 6,35 & 5,59 & 5,58 & 4,89 & 5,81 & 5,58 & 1581 & 1583 \\
\hline Globulol & $\operatorname{tr}$ & $\operatorname{tr}$ & tr & $\operatorname{tr}$ & $\operatorname{tr}$ & $\operatorname{tr}$ & 1589 & 1590 \\
\hline Khusimona & 2,31 & 2,27 & 2,22 & 1,82 & 2,27 & 2,59 & 1600 & 1604 \\
\hline$\beta$-oplopenona & 1,12 & 0,80 & 0,77 & 0,64 & 0,72 & 0,71 & 1606 & 1607 \\
\hline N.I.* & 0,59 & 0,66 & 0,67 & 0,56 & 0,60 & 0,73 & 1634 & - \\
\hline N.I. * & 0,69 & $\operatorname{tr}$ & $\operatorname{tr}$ & $\operatorname{tr}$ & $\operatorname{tr}$ & 0,61 & 1625 & - \\
\hline epi- $\alpha$-cadinol & 1,88 & 2,03 & 2,27 & 1,97 & 2,28 & 2,40 & 1638 & 1640 \\
\hline$\alpha$-murolol & $\operatorname{tr}$ & 0,70 & $\operatorname{tr}$ & $\operatorname{tr}$ & $\operatorname{tr}$ & $\operatorname{tr}$ & 1642 & 1646 \\
\hline Cubenol & 2,49 & 2,46 & 2,99 & 2,41 & 2,93 & 2,86 & 1638 & 1640 \\
\hline 14-hidroxi-(z) cariofileno & $\operatorname{tr}$ & $\operatorname{tr}$ & $\operatorname{tr}$ & $\operatorname{tr}$ & 0,63 & $\operatorname{tr}$ & 1666 & 1667 \\
\hline eudesma-4(15),7-dien-1 $\beta$-ol & 1,09 & 1,23 & 1,39 & 1,34 & 1,26 & 1,37 & 1682 & 1688 \\
\hline
\end{tabular}

${ }^{*}$ N.I.: substância não identificada pelas bibliotecas consultadas e índice de retenção. IRcal = índice de retenção de Kovats calculado; IRlit = índice de retenção de Kovats da literatura; $\operatorname{tr}<0.4$.

diferença significativa para o teste Tukey a $5 \%$ de probabilidade $(p<0,05)$, entre as doses avaliadas (Tabela 2) e foi observada a mesma tendência que na produtividade do componente $\gamma$-muroleno (Figura 3), entretanto menos acentuada. Conforme o aumento das aplicações do composto orgânico houve incremento na produtividade de $0,11 \mathrm{~kg} \mathrm{ha}^{-1}$ a mais que a testemunha $\left(0 \mathrm{t} \mathrm{ha}^{-1}\right)$, atingindo $0,20 \mathrm{~kg} \mathrm{ha}^{-1}$ quando utilizada $30 \mathrm{t} \mathrm{ha}^{-1}$ de composto orgânico.
Quando aplicados 40 e 50 tha-1 $^{-1}$ de composto orgânico a tendência da produtividade, deste componente, é decrescente. Compostos voláteis sesquiterpênicos possuem atividades antibacterianas, fungicidas e inseticidas e com isso este resultado pode ser importante para a produção deste composto.

$\mathrm{Na}$ produtividade do componente sesquiterpênico $\delta$-cadineno (Figura 4 ) verificou-se diferença estatística significativa para o teste Tukey 


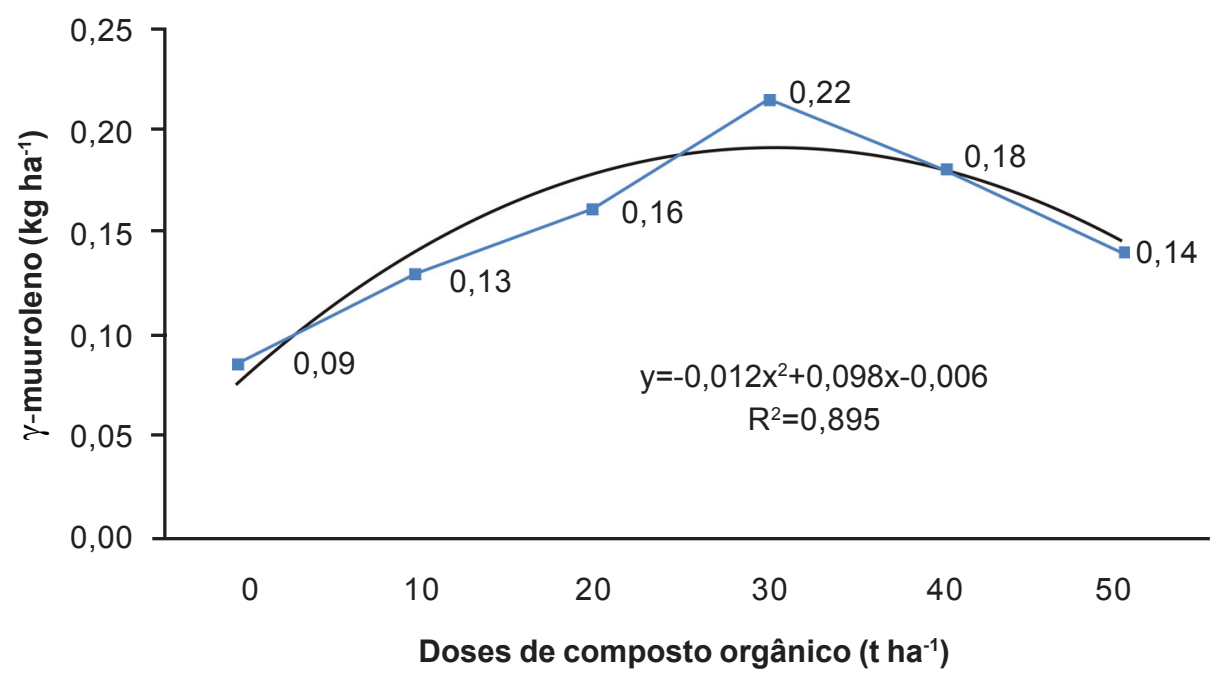

FIGURA 2. Produtividade em $\mathrm{kg} \mathrm{ha}^{-1}$ do componente $\gamma$-muuroleno, do óleo essencial de $B$. dracunculifolia, avaliadas em doses crescentes de composto orgânico: 0, 10, 20, 30, 40 e 50 t ha-1 $^{-1}$, aos 150 DAT (dias após o transplante), cultivadas em Botucatu, SP.

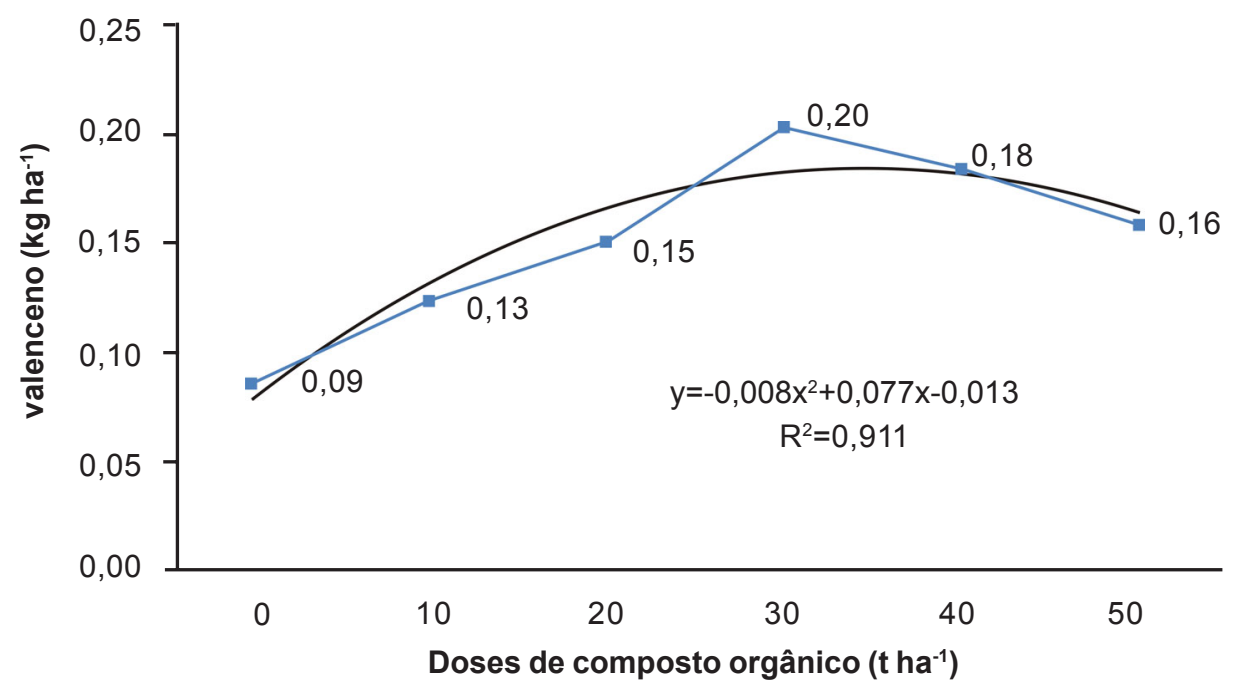

FIGURA 3. Produtividade em kg ha-1 do componente valenceno, do óleo essencial de $B$. dracunculifolia, avaliadas em doses crescentes de composto orgânico: 0, 10, 20, 30, 40 e 50 t ha-1 ${ }^{-1}$ aos 150 DAT (dias após o transplante), cultivadas em Botucatu, SP.

a $5 \%$ de probabilidade $(p<0,05)$, entre as doses avaliadas (Tabela 2) e através da análise de regressão observou-se o aumento gradativo na produtividade deste composto com o acréscimo da dose de composto orgânico, sendo que a dose avaliada que proporcionou maior produtividade foi a de $30 \mathrm{t} \mathrm{ha}^{-1}$ com 0,15 kg ha-1, incremento de 0,09 kg ha-1 em relação à testemunha, e que o aumento da adubação a partir desse ponto causou diminuição deste componente.

Este composto é precursor do gossipol composto que atua na defesa química das plantas e este resultado pode ser importante caso haja interesse na produção deste composto.

O sesquiterpeno $E$-nerolidol apresentou o percentual mais elevado em relação aos demais de 32,06 a 37,57 \% (Tabela 2), diferindo estatisticamente a $5 \%$ de probabilidade para o teste Tukey $(p<0,05)$ (Tabela 2) e a dose $30 \mathrm{t} \mathrm{ha}^{-1}$ apresentou o melhor resultado com $1,56 \mathrm{~kg} \mathrm{ha}^{-1}$, sendo $1,05 \mathrm{~kg} \mathrm{ha}^{-1}$ superior a dose $0 \mathrm{t} \mathrm{ha}^{-1}$ que apresentou $0,51 \mathrm{~kg} \mathrm{ha}^{-1}$ deste componente (Figura 5).

Este resultado é importante tendo em vista suas características antineoplásicas, inibitória do 


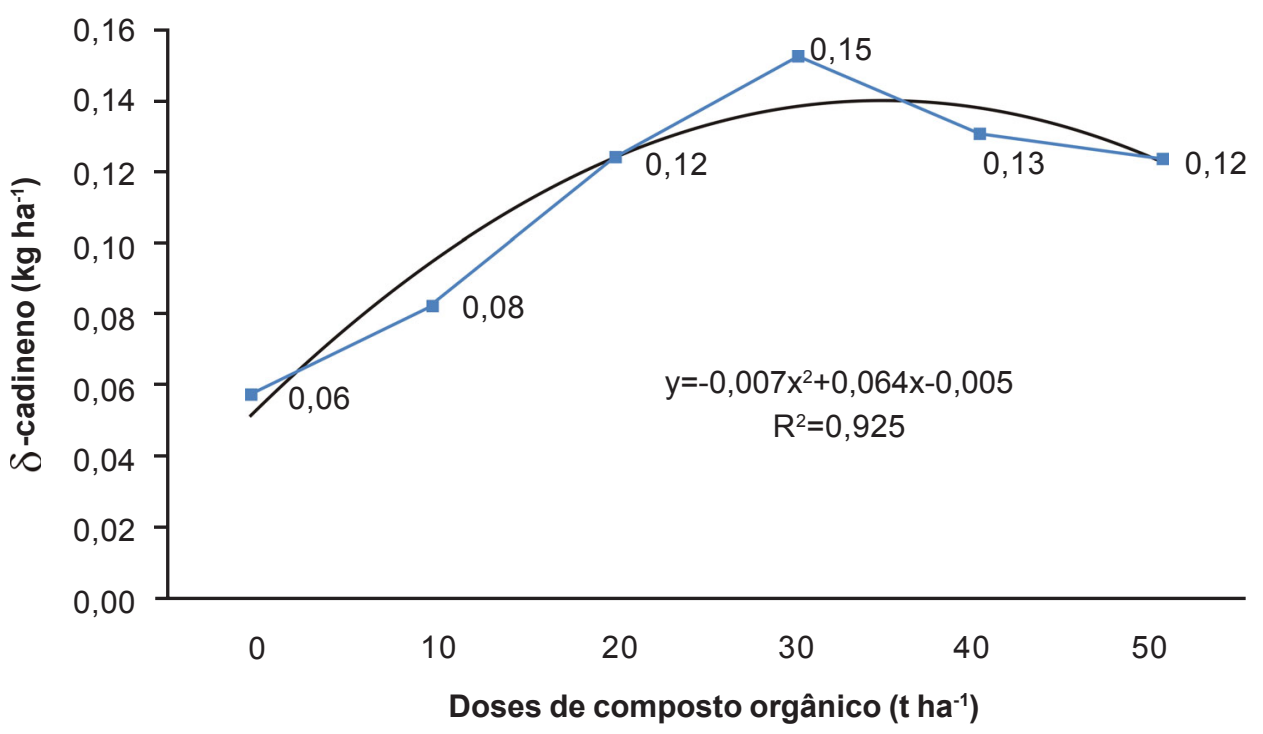

FIGURA 4. Produtividade em $\mathrm{kg} \mathrm{ha}^{-1}$ do componente $\delta$-cadineno, do óleo essencial de $B$. dracunculifolia, avaliadas em doses crescentes de composto orgânico: 0, 10, 20, 30, 40 e 50 t ha-1 $^{-1}$, aos 150 DAT (dias após o transplante), cultivadas em Botucatu, SP.

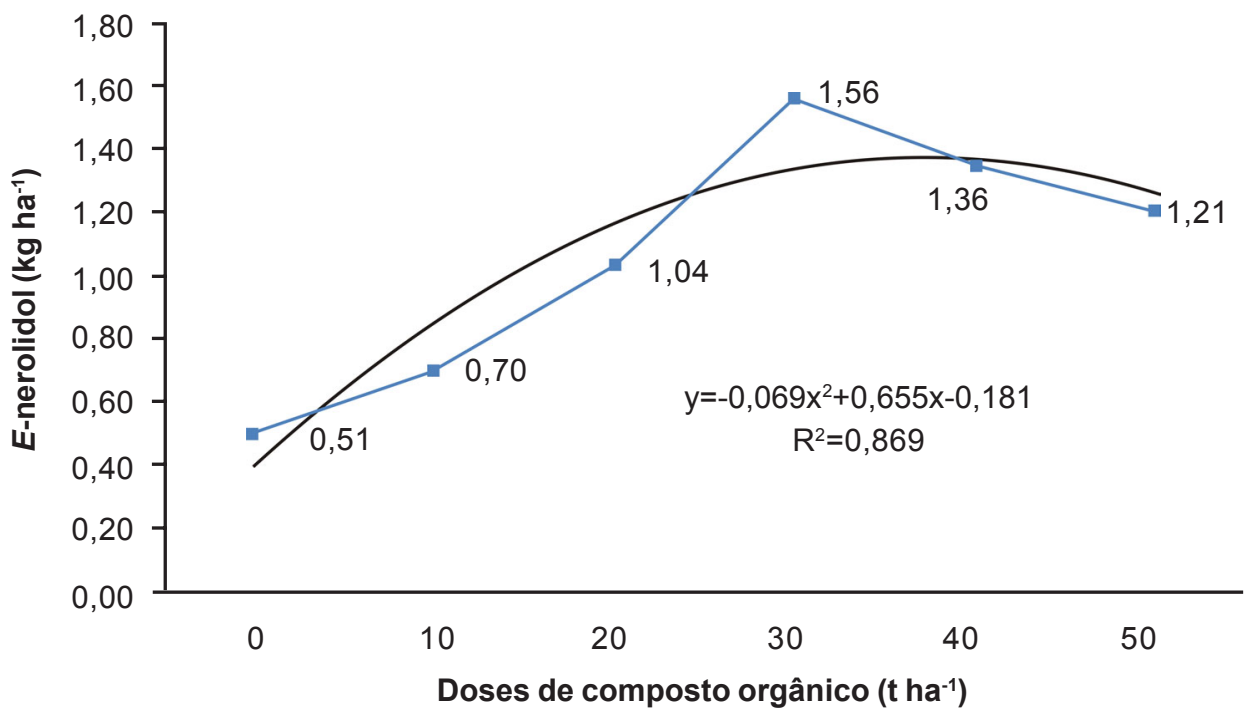

FIGURA 5. Produtividade em kg ha-1 do componente $E$-nerolidol, do óleo essencial de $B$. dracunculifolia, avaliadas em doses crescentes de composto orgânico: 0, 10, 20, 30, 40 e 50 t ha-1 $^{-1}$, aos 150 DAT (dias após o transplante), cultivadas em Botucatu, SP.

crescimento do agente causador da malária e do agente causador da leishmaniose tegumentar americana, de grande interesse para a indústria alimentícia, por suas características flavorizantes, para a indústria de fragrâncias, pois o óleo essencial da $B$. dracunculifolia DC. é considerado perfume exótico e fixador natural, fundamental na composição dos perfumes, sendo exportado como matéria-prima para confecção de perfumes, como este composto é componente majoritário do óleo essencial, isso pode indicá-lo como o maior responsável pelas propriedades odoríficas intensas.

Para o componente sesquiterpênico espatulenol houve diferença estatística significativa, para o teste Tukey a $5 \%$ de probabilidade, $(p<0,05)$, (Tabela 2) com os melhores resultados para as doses 30 e $40 \mathrm{t} \mathrm{ha}^{-1} \mathrm{com} 0,72 \mathrm{~kg} \mathrm{ha}^{-1}$ sendo $0,37 \mathrm{~kg} \mathrm{ha}^{-1}$ superior a dose 0 t ha- $^{-1}$ ( Figura 6 ).

Para a indústria de fragrâncias este resultado é relevante pois sendo este composto componente majoritário também pode ser o responsável pelas propriedades odoríficas intensas. Se o objetivo for este 


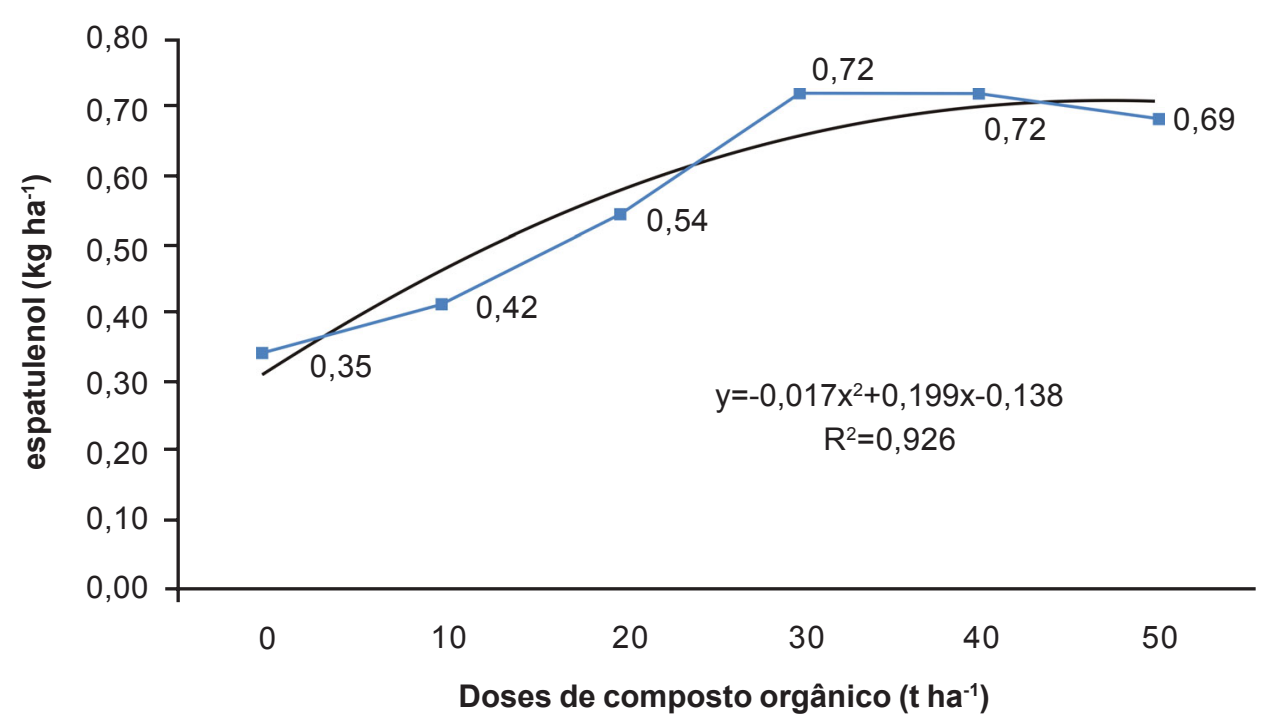

FIGURA 6. Produtividade em kg ha-1 do componente espatulenol, do óleo essencial de $B$. dracunculifolia, avaliadas em doses crescentes de composto orgânico: 0, 10, 20, 30, 40 e 50 t ha-1 $^{-1}$, aos 150 DAT (dias após o transplante), cultivadas em Botucatu, SP.

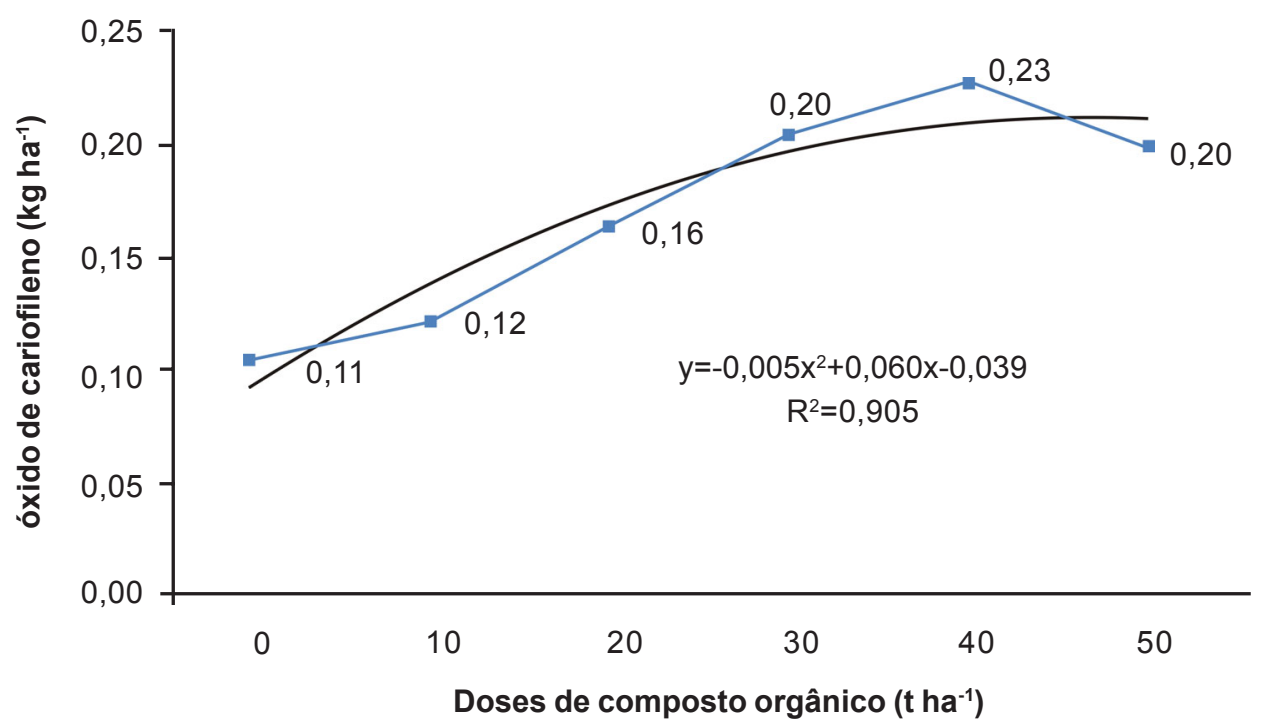

FIGURA 7. Produtividade em kg ha-1 do componente óxido de cariofileno, do óleo essencial de $B$. dracunculifolia, avaliadas em doses crescentes de composto orgânico: 0, 10, 20, 30, 40 e 50 t ha-1 aos 150 DAT (dias após o transplante), cultivadas em Botucatu, SP.

componente a adubação na dose $30 \mathrm{t} \mathrm{ha}^{-1}$ é recomedada, e esta informação é relevante para o produtor rural.

A produtividade do sesquiterpeno óxido de cariofileno apresentou o melhor resultado com $0,23 \mathrm{~kg}$ ha-1 quando utilizada a dose $40 \mathrm{t} \mathrm{ha}^{-1}$, sendo $0,12 \mathrm{~kg}$ ha $^{-1}$ superior a dose 0 tha-1 (Figura 7). Houve diferença estatística significativa, para o teste Tukey a $5 \%$ de probabilidade $(p<0,05)$ (Tabela 3$)$. Este componente possui atividade anticarcinogênica e este resultado é de grande importância para a indústria farmacêutica.
No óleo essencial de alecrim-do-campo $(B$. dracunculifolia) 28 compostos estão presentes, sendo o E-nerolidol e o espatulenol os compostos majoritários.

Os três componentes sesquiterpênicos, $E$ nerolidol, espatulenol e óxido de cariofileno, perfazem $58,44 \%$ da média relativa da composição química do óleo essencial de $B$. dracunculifolia, evidenciando a importância destes e consequentemente a importância dos resultados discutidos anteriormente. Os três componentes $\gamma$-muuroleno, valenceno e $\delta$ cadineno perfazem $14,12 \%$ da média relativa da 
TABELA 3. Médias (teste Tukey) de produtividade em $\mathrm{kg} \mathrm{ha}^{-1}$ dos componentes do óleo essencial de $B$. dracunculifolia avaliadas em doses crescentes de composto orgânico: 0, 10, 20, 30, 40 e 50 t ha- $^{-1}$, aos 150 DAT (dias após o transplante), cultivadas em Botucatu, SP.

\begin{tabular}{ccccccc}
\hline Doses & $\gamma$-muuroleno & valenceno & $\delta$-cadineno & E-nerolidol & espatulenol & óxido de cariofileno \\
\hline 0 & $0,09 \mathrm{c}$ & $0,09 \mathrm{c}$ & $0,06 \mathrm{c}$ & $0,51 \mathrm{c}$ & $0,35 \mathrm{~b}$ & $0,11 \mathrm{~b}$ \\
10 & $0,13 \mathrm{bc}$ & $0,13 \mathrm{bc}$ & $0,08 \mathrm{bc}$ & $0,70 \mathrm{bc}$ & $0,42 \mathrm{ab}$ & $0,12 \mathrm{ab}$ \\
20 & $0,16 \mathrm{ab}$ & $0,15 \mathrm{abc}$ & $0,12 \mathrm{ab}$ & $1,04 \mathrm{abc}$ & $0,54 \mathrm{ab}$ & $0,16 \mathrm{ab}$ \\
30 & $0,22 \mathrm{a}$ & $0,20 \mathrm{a}$ & $0,15 \mathrm{a}$ & $1,56 \mathrm{a}$ & $0,72 \mathrm{a}$ & $0,20 \mathrm{ab}$ \\
40 & $0,18 \mathrm{ab}$ & $0,18 \mathrm{ab}$ & $0,13 \mathrm{ab}$ & $1,36 \mathrm{ab}$ & $0,72 \mathrm{a}$ & $0,23 \mathrm{a}$ \\
50 & $0,14 \mathrm{bc}$ & $0,16 \mathrm{abc}$ & $0,12 \mathrm{ab}$ & $1,21 \mathrm{abc}$ & $0,69 \mathrm{ab}$ & $0,20 \mathrm{ab}$ \\
\hline $\mathbf{P}$ & $\mathbf{0 , 0 0 1}$ & $\mathbf{0 , 0 0 1 7}$ & $\mathbf{0 , 0 0 1 1}$ & $\mathbf{0 , 0 0 6 8}$ & $\mathbf{0 , 0 1 5 2}$ & $\mathbf{0 , 0 2 5 6}$ \\
\hline $\mathbf{C V}(\%)$ & $\mathbf{2 1 , 1 7}$ & $\mathbf{2 0 , 4 2}$ & $\mathbf{2 2 , 3 4}$ & $\mathbf{3 3 , 5 7}$ & $\mathbf{2 7 , 1 9}$ & $\mathbf{2 9 , 7 2}$ \\
\hline
\end{tabular}

Médias seguidas de letras iguais na coluna não diferiram estatisticamente entre si pelo Teste de Tukey $(p>0,05)$.

composição química do óleo essencial de $B$. dracunculifolia. Somados aos compostos E-nerolidol, espatulenol e óxido de cariofileno prefazem o total de $72,56 \%$ da média relativa da composição química do óleo essencial de $B$. dracunculifolia, composto pela presença de 28 substâncias.

$\mathrm{Na}$ produtividade dos componentes $\gamma$ muuroleno, valenceno, $\delta$-cadineno e nerolidol as dosagens estudadas influenciaram as plantas, que na dosagem $30 \mathrm{tha}^{-1}$ obtiveram os melhores resultados. Se o objetivo no cultivo de $B$. dracunculifolia for o componente espatulenol as dosagens 30 e 40 t ha $^{-1}$ obtiveram os melhores resultados. Para a produtividade do componente óxido de cariofileno as dosagens estudadas influenciaram as plantas, que na dosagem 40 t ha- $^{-1}$ obtiveram os melhores resultados.

Conclui-se que a técnica de CG/EM proporcionou a identificação de vinte e quatro compostos químicos no óleo essencial, entre monoterpenos e sesquiterpenos. Os compostos majoritários do óleo essencial foram o E-nerolidol e o espatulenol. A partir dos resultados da produtividade do óleo essencial, obtido aos 150 dias após o transplante das mudas, permitese a projeção de uma produção de $0,22 \mathrm{Kg} \mathrm{ha}^{-1}$ do componente $\gamma$-muuroleno, de $0,20 \mathrm{Kg} \mathrm{ha}^{-1}$ do componente valenceno, de $0,15 \mathrm{Kg} \mathrm{ha}^{-1}$ do componente $\delta$-cadineno, e $1,56 \mathrm{Kg} \mathrm{ha}^{-1}$ do componente $E$-nerolidol, todos na dosagen $30 \mathrm{t} \mathrm{ha}^{-1}$ de composto orgânico, de $0,72 \mathrm{Kg} \mathrm{ha}^{-1}$ do componente espatulenol obtida nas dosagens $30 \mathrm{e}$ 40 t ha-1 $^{-1}$ de composto orgânico e produção de 0,23 $\mathrm{kg} \mathrm{ha}^{-1}$ do componente óxido de cariofileno na dose 40 tha $^{-1}$ de composto orgânico.

Ao observar as respostas das plantas às doses crescentes de composto orgânico, deve-se considerar além da produção de fitomassa, principalmente, a produção dos teores de princípios ativos, bem como os fatores econômicos e com isso a dose 30 t ha $^{-1}$ é a recomendação, para os resultados deste estudo, com exceção do componente óxido de cariofileno que obteve o melhor resultado na dose $40 \mathrm{t} \mathrm{ha}^{-1}$.

\section{REFERÊNCIA}

ADAMS, R.P. Identification of essential oil components by gas cromatography/mass spectroscopy. Carol Stream: Allured Publishing Corporation, 2001. 451p. ARAÚJO, A.M.; FERNANDES, G.W.; BEDÊ, L.C. Influência do sexo e fenologia de Baccharis dracunculifolia DC. (Asteraceae) sobre insetos herbívoros. Revista Brasileira de Entomologia, v.39, n.2, p.347-53, 1995.

AZEVEDO, S.K.S.; SILVA, I.M. Plantas medicinais e de uso religioso comercializadas em mercados e feiras livres no Rio de Janeiro - RJ, Brasil. Acta Botanica Brasilica, v.20, n.1, p.185-94, 2006.

BANZATTO, D.A.; KRONKA, S.N. Experimentação agrícola. 3.ed. Jaboticabal: Funep,1995. 247p.

CAMBRAIA, J. Estresses ambientais danos e benefícios em plantas. Recife: UFRPE Imprensa Universitária, 2005. 500p.

COLEVATTI, R.G.; SPERBER, C.F. The gall maker Neopelma baccharis Burck. (Homoptera: Psyllidae) on Baccharis dracunculifolia DC. (Asteraceae): individual, local, and regional patterns. Anais da Sociedade Entomológica do Brasil, v.26, n.1, p.45-53, 1997.

COSTA, A.G.L.C. Estudo químico de Baccharis dracunculifolia DC. e sua correlação com a própolis de uma microregião de Campos Gerais do Paraná. 2009. 74p. Dissertação (Mestrado - Área de Concentração em Química) - Universidade Estadual de Ponta Grossa, Ponta Grossa.

CUNHA, A.R. et al. Classificação climática para o município de Botucatu, SP, segundo Köppen. In: SIMPÓSIO EM ENERGIA NA AGRICULTURA, 1., 1999, Botucatu. Anais... Botucatu: Faculdade de Ciências Agronômicas, 1999. p.490-1.

DUARTE, S. et al. Effect of a novel type of propolis and its chemical fractions on glucosyltransferases and on growth and adherence of mutans streptococci. Biological 
\& Pharmaceutical Bulletin, v.26, n.4, p.527-31, 2003.

EMBRAPA. Centro Nacional de Pesquisa de Solos. Sistema brasileiro de classificação de solos. 2.ed. Rio de Janeiro: EMBRAPA Solos, 2006. 306p.

ESPIRITO SANTO, M.M. et al. Tannins in Baccharis dracunculifolia (Asteraceae): effects of seasonality, water availability and plant sex. Acta Botanica Brasilica, v.13, n.2, p.167-74, 1999.

FENNER, R. et al. Plantas utilizadas na medicina popular brasileira com potencial atividade antifúngica. Revista Brasileira de Ciências Farmacêuticas, v.42, n.3, p.36974, 2006. Disponível em: <http://www.scielo.br/pdf/rbcf/ v42n3/a07v42n3.pdf>. Acesso em: 14 mai. 2011.

FERRACINI, V.L. et al. Essential oils of seven brazilian Baccharis species. The Journal of Essential Oil Research, v.7, p.355-67, 1995.

FUKUDA, M. et al. Studies on the constituents of the leaves of Baccharis dracunculifolia (Asteraceae) and their cytotoxic activity. Pharmaceutical Society of Japan, v.54, n.10, p.1465-8, 2006.

LOPES, A.S.; GUIMARÃES, P.T.G. Recomendações para o uso de corretivos e fertilizantes em Minas Gerais: 4a aproximação. Lavras: CFSEMG, 1989. p.176.

MAPA. Ministério da Agricultura, Pecuária e Abastecimento. Manual de métodos analíticos oficiais para fertilizantes minerais, orgânicos, organominerais e corretivos. Brasília: MAPA, 2007. 141p.

MCLAFFERTY, F.W.; STAUFFER, D. The wile/NBS registry of mass spectral data. New York: John Wiley \& Sons, 1989. v.1-6.

MARTINS, E.R. et al. Plantas medicinais. Viçosa: UFV, 1998. 220p.

QUEIROGA, C.L. Estudo fitoquímico do óleo essencial de Baccharis dracunculifolia. 1989. 193p. Dissertação (Mestrado - Área de Concentração em Química) Universidade Estadual de Campinas, Campinas.

RODRIGUES, V.E.G.; CARVALHO, D.A.D.E. Levantamento etnobotânico de plantas medicinais no domínio do Cerrado na região do alto Rio Grande - Minas Gerais. Ciência e Agrotecnologia, v.25, n.1, p.102-23, 2001.

VAN DEN DOOL, H.; KRATZ, D.J. A generalization of the relation index system including liner temperature programmed gas-liquid partition chromatography. Journal of Chromatography, v.11, p.463-7, 1963. 